\title{
Integração de tecnologias digitais no ensino de enfermagem: criação de um caso clínico sobre úlceras por pressão com o software SIACC
}

\author{
Integration of digital technologies in nursing teaching: simulation of a \\ clinical case about pressure ulcers with the SIACC software
}

\section{La integración de las tecnologías digitales en la educación de enfermería: simulación de un caso clínico sobre úlcera de decúbito con el software SIACC}

Luzia Fernandes Millão | luziam.ufcspa@gmail.com

Universidade Federal de Ciências da Saúde de Porto Alegre (UFCSPA). Porto Alegre, Brasil.

Tainara Wink Vieira | tainara.winkv@gmail.com

Universidade Federal de Ciências da Saúde de Porto Alegre (UFCSPA). Porto Alegre, Brasil.

Natália Domingues dos Santos | ndominguesody@gmail.com

Universidade Federal de Ciências da Saúde de Porto Alegre (UFCSPA). Porto Alegre, Brasil.

Ana Paula Scheffer Schell da Silva | anaschell@ufcspa.edu.br

Universidade Federal de Ciências da Saúde de Porto Alegre (UFCSPA). Porto Alegre, Brasil.

Cecília Dias Flores | dfflores@gmail.com

Universidade Federal de Ciências da Saúde de Porto Alegre (UFCSPA). Porto Alegre, Brasil.

\section{Resumo}

A atenção à saúde exige cada vez mais profissionais com habilidades de pensamento crítico-reflexivo e tomada de decisões, constituindo um desafio aos educadores a implementação de metodologias ativas de aprendizagem condizentes com o avanço tecnológico atual. Dessa forma, o intuito deste artigo é apresentar o desenvolvimento de simulação de um caso clínico por meio do software SIACC (Sistema Interdisciplinar de Análise de Casos Clínicos) para auxiliar o estudo de fundamentos de enfermagem. O processo de criação do caso clínico no SIACC foi dividido em duas etapas: cadastro de informações e montagem do caso. O casopiloto desenvolvido sobre 'úlceras por pressão' apresenta imagens, vídeos, artigos e exercícios para facilitar o aprendizado do aluno sobre o tema abordado. Os resultados apresentados reforçam que a utilização de simulação clínica virtual no ensino dessa profissão favorece a correlação entre as atividades teóricas e as experiências clínicas, auxiliando o aluno no desenvolvimento de conhecimentos, habilidades e atitudes fundamentais para o exercício do cuidado de enfermagem.

Palavras-chave: educação em enfermagem; tecnologia educacional; simulação por computador; ensino; enfermagem. 


\begin{abstract}
The health care demands more and more professionals with reflexive critical thinking and decision-making habilities. Therefore, is a challenge to educators to implement active learning methodologies that are befitting with the technology development today. Thus, the goal of this article is to present the development of a simulation of a clinical case using the software titled SIACC (Sistema Interdisciplinar de Análise de Casos Clínicos - interdisciplinary system for analysis of clinical cases) to improve the study of nursing fundamentals. The process to simulate clinical case with the SIACC was divided in two steps: information register and the case setting in the software. The pilot project about "pressure ulcers" includes pictures, videos, articles and exercises to facilitate the learning of the theme by the student. The results ratify that the use of clinical simulation through that software in nursing education facilitates the correlation between theory and clinical experiences, enabling the student to develop knowledge, skills and attitudes that are fundamental to nursing care.
\end{abstract}

Keywords: nursing education; educational technology; computer simulation; teaching; nursing.

\title{
Resumen
}

La atención de salud requiere cada vez más profesionales con habilidades de pensamiento crítico y reflexivo y toma de decisiones, siendo un desafío para los educadores aplicar metodologías de aprendizaje activo en consonancia con el avance tecnológico actual. El objetivo de este artículo es presentar el desarrollo de una simulación de un caso clínico con utilización del software SIACC (Sistema Interdisciplinar de Análise de Casos Clínicos - sistema interdisciplinario de casos de análisis clínico) para ayudar al estudio de los fundamentos de enfermería. El proceso de creación del caso en SIACC se dividió en dos etapas: el registro de informaciones y el montaje de caso clínico. El caso piloto acerca de las "úlceras de decúbito" muestra imágenes, videos, artículos y ejercicios para facilitar el aprendizaje, por los estudiantes, del tema tratado. Los resultados que se presentan confirman que el uso de la simulación clínica virtual en la educación de enfermería favorece la correlación entre las actividades teóricas y experiencias clínicas, ayudando a los estudiantes a desarrollar conocimientos, habilidades y actitudes esenciales para el ejercicio de los cuidados de enfermería.

Palavras-clabe: educación em enfermeira; tecnologia educacional; simulación por computador; enseñanza, enfermeira.

Contribuição dos autores: As autoras declaram ter contribuído em todas as fases de produção do artigo.

Declaração de conflito de interesses: As autoras declaram não haver qualquer conflito de interesse.

Fontes de financiamento: Pró-Reitoria de Graduação da Universidade Federal de Ciências da Saúde de Porto Alegre (PROGRAD UFCSPA), através do Programa de Iniciação à Docência (PID) da UFCSPA.

Considerações éticas: $O$ presente artigo trata-se de um relato de experiência acerca da formulação de casos clínicos em um sistema web, de forma que não envolveu riscos a seres humanos ou animais, estando, portanto, em conformidade com a resolução no 466/2012 do Ministério da Saúde e Conselho Nacional de Saúde.

Agradecimento/Contribuições adicionais: À UFCSPA, pelo incentivo financeiro para realização de tal trabalho; e aos autores do SIACC.

Histórico do artigo: Submetido: 09.set.2016 | Aceito: 01.dez.2016 | Publicado: 31.mar.2017

Apresentação anterior: 0 artigo não foi apresentado anteriormente.

Licença CC BY-NC atribuição não comercial. Com essa licença é permitido acessar, baixar (download), copiar, imprimir, compartilhar, reutilizar e distribuir os artigos, desde que para uso não comercial e com a citação da fonte, conferindo os devidos créditos de autoria e menção à Reciis. Nesses casos, nenhuma permissão é necessária por parte dos autores ou dos editores. 


\section{Introdução}

Há alguns anos, o paradigma da transmissão vertical de conhecimento vem se rompendo, fazendo pensar no processo de ensino-aprendizagem no qual o aluno passa a ser sujeito da (re) construção do saber, formulador de hipóteses, pesquisador, capaz de tomar decisões e estar continuamente buscando a sua atualização ${ }^{1}$. Assim, passa a ser sujeito da aprendizagem, o que significa que ele mesmo busca os conhecimentos necessários para responder a uma pergunta, a um problema e a uma situação².

Essa nova maneira de problematizar a educação e a relação com o conhecimento confere ao estudante o papel central em seu processo educacional e preconiza a interatividade entre os professores, tutores e estudantes como um dos pilares para a educação a distância de qualidade. Entretanto, para tal, é necessário que haja um sistema de comunicação que permita a resolução rápida de problemas relacionados ao material e ao conteúdo, bem como aspectos relativos à orientação de aprendizagem como um todo.

As instituições de ensino superior (IES) têm sido motivadas a refletir e implementar mudanças em suas grades curriculares, de forma que sejam adotadas alternativas inovadoras de ensino que priorizem metodologias ativas, definindo um aprendizado que facilite a aquisição de habilidades, competências e atitudes baseadas em resultados ${ }^{3}$.

Nesse contexto, entende-se que os cenários de aprendizagem devem incorporar e interligar métodos didático-pedagógicos; de áreas de práticas e vivências; de utilização de tecnologias e habilidades cognitivas e psicomotoras; de valorização dos preceitos morais e éticos, orientadores de condutas individuais e coletivas; e de organização do processo de trabalho4.

No âmbito da saúde, a discussão sobre a adoção de metodologias ativas de aprendizagem tomou contornos próprios, na medida em que a forma de ensino da teoria e sua integração com a prática é aprimorada a cada dia, a fim de formar profissionais com uma visão integral e holística do cuidado.

As Diretrizes Curriculares Nacionais para os cursos de enfermagem no país preconizam a formação desse profissional generalista, humanista, crítico e reflexivo, qualificado para intervir em situações de saúde e doença prevalentes no quadro epidemiológico nacional ${ }^{5}$.

Além disso, o mercado de trabalho demanda profissionais que estejam habilitados a lidar com as tecnologias emergentes, de forma que os modelos tradicionais de ensino já não comportam tamanha quantidade de informação.

A ressignificação do processo de ensino-aprendizagem é resultado também do intenso desenvolvimento das tecnologias de informação e comunicação, que legitimam o caráter dinâmico e urgente do conhecimento nos dias atuais e suscitam discussões quanto às novas modalidades educacionais e suas potencialidades, dentre as quais se destaca a educação a distância ${ }^{6}$.

A difusão do acesso à internet possibilita a adoção de novas abordagens no ensino voltados para a saúde ${ }^{7}$. Dessa forma, as ferramentas computacionais contribuem para suprir as demandas de informações complexas e em grande quantidade que são imprescindíveis para os processos de tomada de decisão, além de fornecer novas formas de material didático que incluem som, imagem e vídeo de forma interativa.

Portanto, a adoção de Tecnologias de Informação e Comunicação (TIC) com base na internet e na modalidade de educação a distância tem se tornado cada vez mais comum na educação em enfermagem. A simulação de pacientes virtuais e os objetos de aprendizagem (OA) são alguns exemplos que devem ser citados. 


\section{Simulação de pacientes virtuais}

A simulação se refere à aplicação de modelos computacionais para estudo e previsão de eventos ou comportamentos, disponibilizada para uma ampla gama de aplicações, sendo utilizada principalmente na área da educação ${ }^{8}$. A simulação clínica, nesse contexto, tenta replicar os aspectos essenciais de situações clínicas, sendo tendência no ensino tanto de alunos de graduação quanto de pós-graduação de cursos de ciências da saúde. Consiste em uma estratégia ativa de aprendizagem centralizada no aprendiz, e que o professor atua como facilitador do aprendizado'.

A simulação no ensino de enfermagem é reconhecida como uma estratégia complementar à vivência clínica, de caráter imersivo e amplamente aceita em diversos locais no mundo. O método é bem reconhecido por preparar os alunos para o grande espectro de desafios clínicos que eles podem enfrentar em sua atuação como enfermeiros, de forma que consiste em uma estratégia ideal para o desenvolvimento, refinamento e ensaio de habilidades no reconhecimento e resposta à deterioração de um paciente em um ambiente seguro $^{10}$. Representa a oportunidade de expor os alunos a cenários críticos e apreciar a natureza imprevisível da prática clínica ${ }^{11}$, de forma que aumenta a segurança e autoconfiança na realização de procedimentos e tomada de decisões ${ }^{9}$.

Além disso, estudos recentes demonstram melhor desempenho de alunos que aprenderam situações através do uso de simuladores virtuais em comparação com aqueles que tiveram apenas aulas expositivas tradicionais ${ }^{9,11,12}$.

\section{Objetos de aprendizagem (OA)}

Os objetos de aprendizagem (OA) são definidos como quaisquer recursos digitais que podem ser reutilizados para auxiliar a aprendizagem e podem ser disponibilizados na web, sob demanda, independentemente do tamanho ${ }^{13}$. Os objetos de aprendizagem são elementos digitais de um novo tipo de instrução, podendo ser reutilizados em diferentes contextos pedagógicos e por várias pessoas ${ }^{13}$.

Os OA se tornaram uma importante ferramenta pedagógico-didática, pois trazem a possibilidade da construção de novos modelos organizativos do currículo. Esses objetos impulsionam a aprendizagem, pois os alunos passam a ter atitudes mais interativas, interventivas e participativas.

\section{Objetivos}

Seguindo a tendência de desenvolvimento e o uso de simuladores no ensino na área da saúde, a Universidade Federal de Ciências da Saúde de Porto Alegre (UFCSPA) tem desenvolvido diversas ferramentas que permitem a criação de simulação de casos clínicos interativos na web, denominadas SIACC (Sistema Interdisciplinar de Análise de Casos Clínicos) e SimDeCS (Simuladores Inteligentes para a Tomada de Decisão em Cuidados de Saúde). Estas ferramentas vêm sendo utilizadas por docentes de diversas disciplinas em diferentes departamentos acadêmicos.

Dessa forma, o objetivo deste artigo é descrever o desenvolvimento de um caso clínico virtual como método de simulação clínica criado na web através do software SIACC, com a finalidade de auxiliar os discentes de enfermagem no aprendizado de semiologia e semiotécnica nas disciplinas que abordam os fundamentos de enfermagem da UFCSPA. Para isso, é apresentado um relato de experiência sobre a criação do caso clínico 'úlceras por pressão'. 


\section{Desenvolvimento do caso clínico: o relato de experiência}

O presente estudo apresenta um relato de experiência acerca das atividades desenvolvidas no projeto "Integração de mídias nos espaços de aprendizagem na Enfermagem: utilizando casos interativos como metodologia de simulação", integrante do Programa de Iniciação à Docência (PID) da UFCSPA, uma instituição pública federal de ensino superior especializada na área da saúde, localizada no extremo sul do Brasil. O PID tem como objetivo proporcionar a realização de atividades que estimulem o desenvolvimento de metodologias inovadoras que contribuam com a melhoria do ensino na graduação a partir de novas práticas e experiências pedagógicas.

O sistema web SIACC, cuja finalidade é o desenvolvimento de casos de estudos para as ciências da saúde, foi utilizado para a elaboração dos casos clínicos interativos para a enfermagem. O SIACC foi desenvolvido como um projeto de mestrado na UFCSPA, em parceria com a Universidade Feevale, integrando as ciências da saúde e as ciências exatas. O sistema possibilita o uso de recursos computacionais em sala de aula, possibilitando a simulação de situações cotidianas na prática clínica ${ }^{14}$.

O SIACC é organizado em: objetivos, anamnese, exame físico, hipóteses diagnósticas e exames, diagnóstico, tratamento e desfecho. Há ainda a opção de exercícios e conteúdos extras (Figura 1).

\section{1 - Dados sobre o caso de estudo}

Dados básicos

Colaboradores

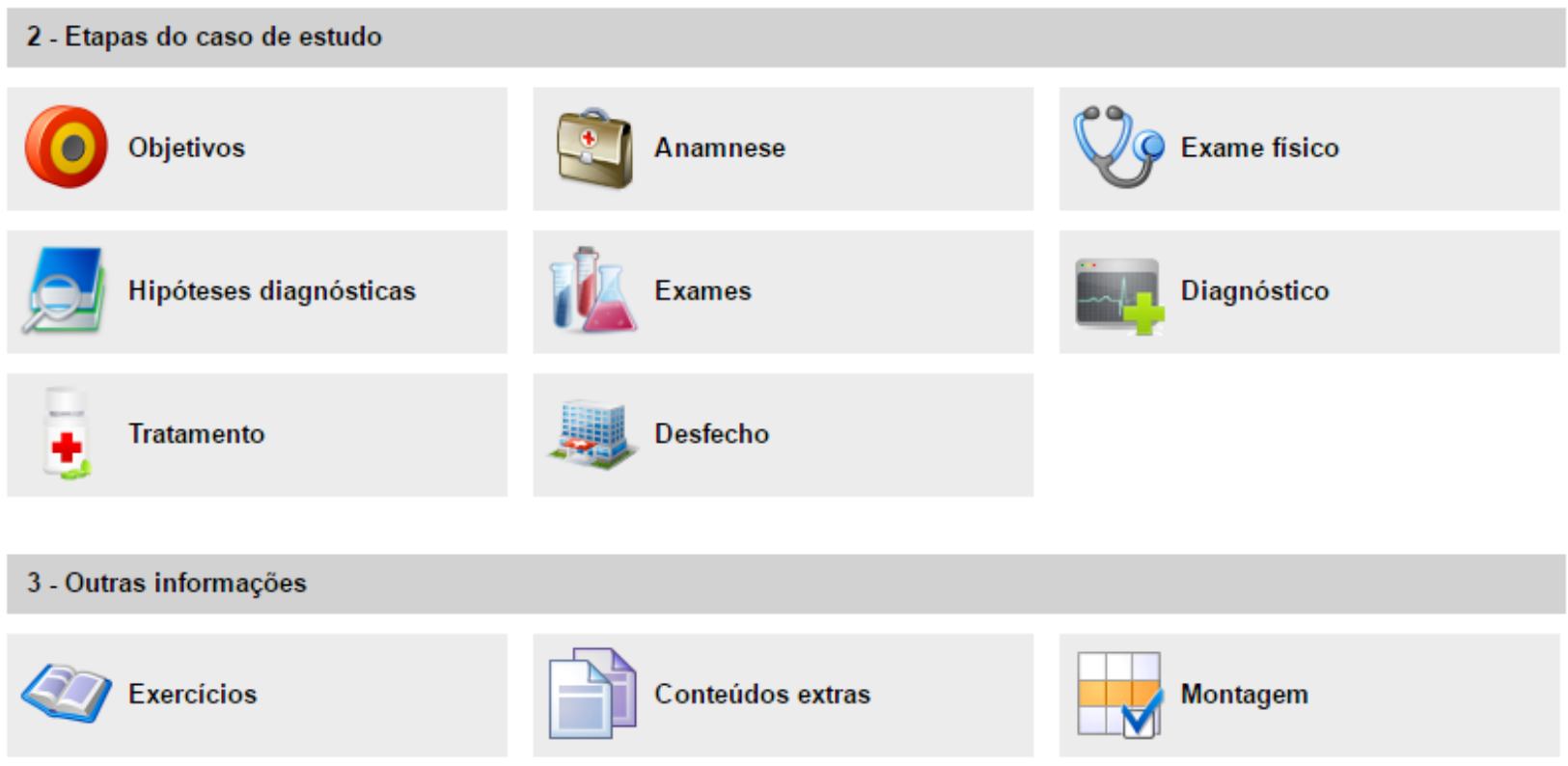

Figura 1 - Tela inicial SIACC

Fonte: SIACC (2016)

O desenvolvimento do caso clínico ocorreu em duas fases: coleta de informações e montagem do caso.

Na primeira etapa foi definido o tema do caso clínico, seguindo os planos de ensino das disciplinas Fundamentos Teóricos de Enfermagem II e Fundamentos para a Prática de Enfermagem I e II. Posteriormente, foi realizada uma pesquisa em bases de dados eletrônicas e livros de fundamentos de enfermagem a fim de organizar o material mais atualizado relacionado ao tema do caso clínico, de forma que o aluno tenha acesso às práticas baseadas em evidências. Os dados relacionados à história do paciente foram 
coletados durante as práticas hospitalares das alunas bolsistas do PID, sem identificação do indivíduo em estudo. Foram incluídos no caso: imagens, vídeos, sons, exercícios de múltipla escolha, artigos científicos e websites para consulta de materiais.

A segunda etapa consistiu na colocação das informações coletadas e organizadas anteriormente no SIACC conforme as etapas do caso de estudo.

Assim, o primeiro tema elencado para elaboração do caso clínico foi 'úlceras por pressão', e este faz a integração entre os conhecimentos teóricos e práticos de semiologia e semiotécnica de enfermagem nos cuidados com a pele e feridas. O caso clínico foi dividido em seções de acordo com as disponibilizadas pelo próprio sistema SIACC, conforme descrito anteriormente. Adaptações à área de enfermagem foram realizadas no material a fim de que pudesse ser melhor aplicável à realidade dos discentes, facilitando a sua compreensão. Tendo em vista que o caso foi o primeiro a ser elaborado no projeto, foi considerado piloto para sua disponibilização aos alunos.

O caso tem início na apresentação dos objetivos, que visam não somente ao ensino da semiotécnica e semiologia de enfermagem, mas também ao processo de enfermagem como um todo, utilizando as taxonomias NANDA-I, NIC (classificações das intervenções de enfermagem) e NOC (classificação dos resultados de enfermagem $)^{15}$.

A Figura 2 apresenta a seção referente à anamnese do paciente em estudo, de forma que essa é dividida em: identificação, queixa principal, história atual, história pregressa, história familiar e perfil psicossocial.

\section{Anamnese (identificação)}

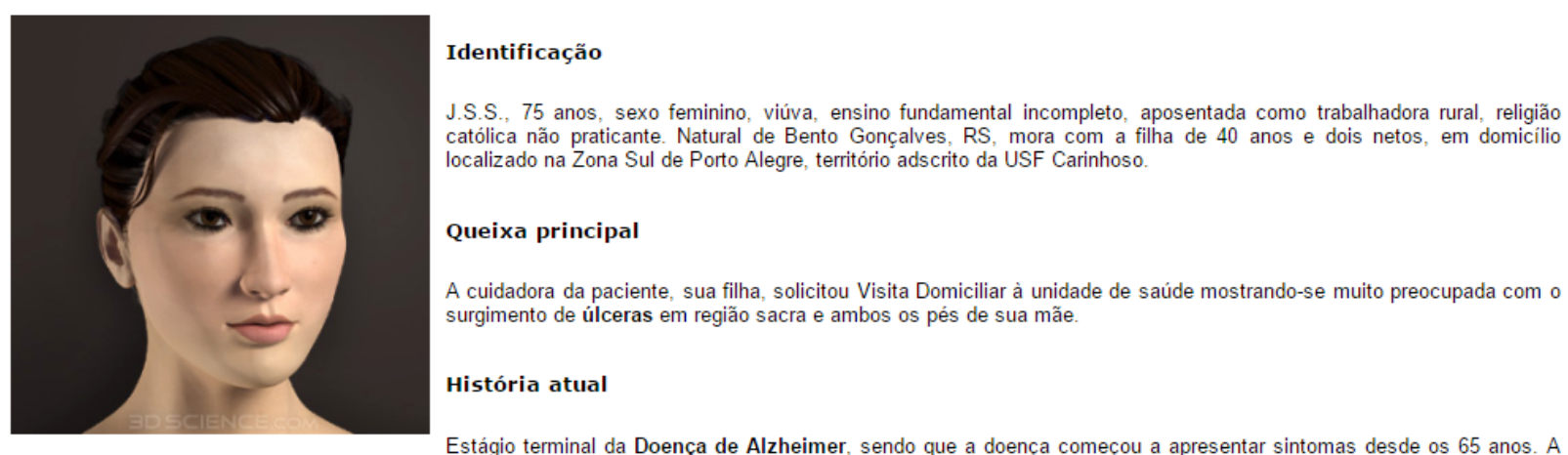
paciente perdeu grande parte da mobilidade física há cerca de três meses, estando acamada desde então. A filha refere que, apesar de cuidar da mãe há anos, não sabe lidar com a situação da mãe acamada, tendo dificuldades em efetuar a troca de decúbito, esperando o marido estar em casa para realizar este cuidado (3x/dia) Além disso, notou o surgimento de úlcera em região sacra e ambos os pés. Alarmada, procurou sobre úlceras na internet e vendo as possiveis complicações, pediu assistência à Unidade A paciente passa a maior parte do tempo dormindo, com períodos de confusão Quanto à alimentação, faz uso de sonda nasoenteral (SNE) assistencia a Unidade. A paciente passa a maior parte do tempo dormindo, com periodos de confusao. Quanto a alimentaçao, faz uso de sonda nasoenteral (SNE) desde janeiro de 2015, última vez que a paciente foi internada no hospital em decorrência de uma pneumonia. A SNE foi colocada pois a paciente já não aceitava mais comida VO, sendo que as refeições deixavam-na agitada e confusa; e perdeu cerca de $8 \mathrm{~kg} \mathrm{em} 2$ semanas. Incontinência urinária, com eliminações em fraldas.
Diabetes Mellitus tipo II, fazendo uso de insulina.

Figura 2 - Anamnese do paciente em estudo no SIACC

Fonte: SIACC (2016)

As Figuras 3, 4 e 5 apresentam as telas da seção de exame físico, na qual consta a imagem de um corpo humano com pontos nos principais locais de exame físico, e o aluno pode clicar naqueles que deseja para obter dados em relação ao exame físico daquele órgão/sistema. Essa seção permite ainda a utilização de áudio, imagens e vídeos, sendo utilizados os sons das bulhas cardíacas, imagens com os pontos anatômicos de maior ocorrência de úlceras por pressão e o estadiamento de feridas, de forma que o estudante esteja apto a compreender os dados recebidos já naquele momento, não exigindo a busca de novas informações. 


\section{Exame Físico}

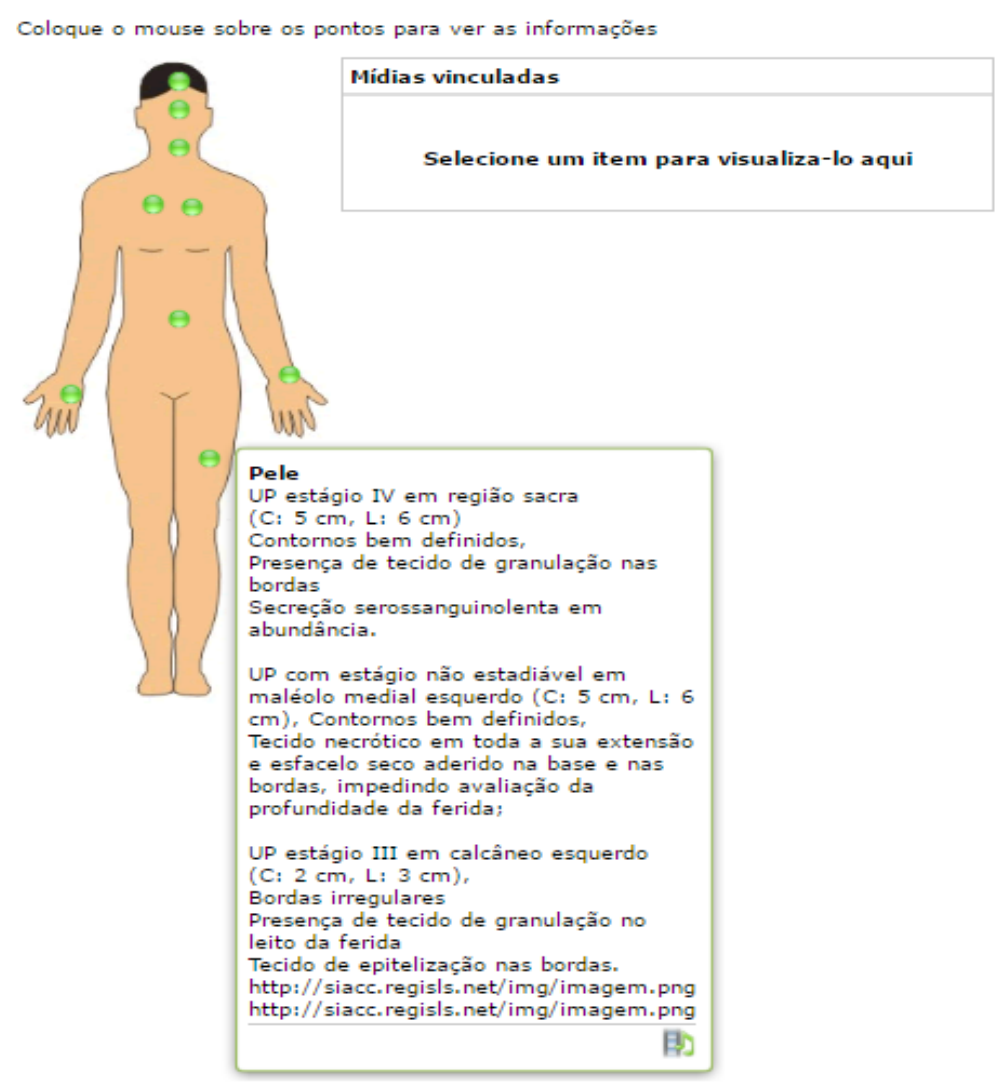

Figura 3 - Exame físico - visualização das informações

Fonte: SIACC (2016)

\section{Exame Físico}

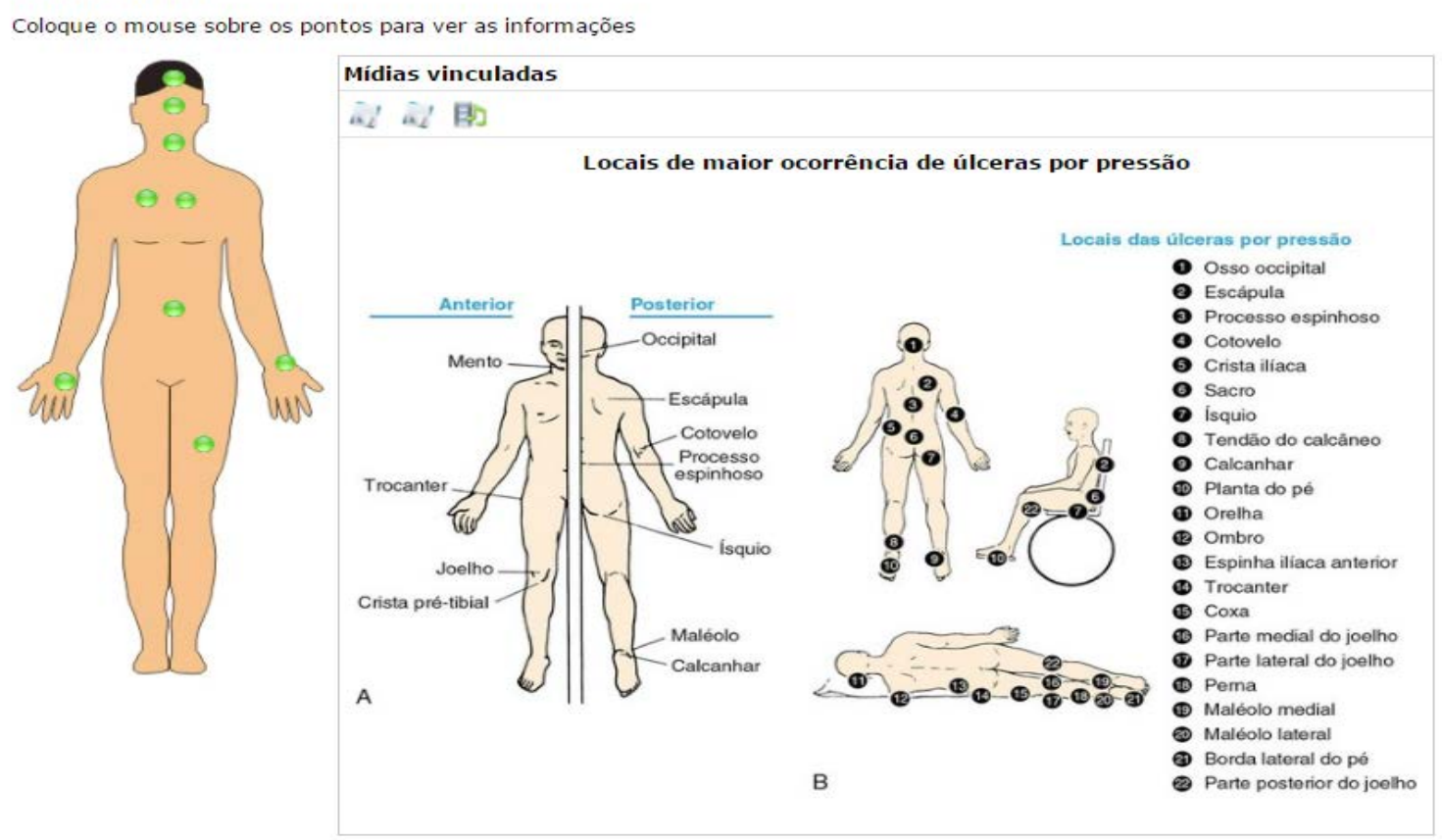

Figura 4 - Utilização de imagens nas informações do exame físico (1)

Fonte: SIACC (2016) 


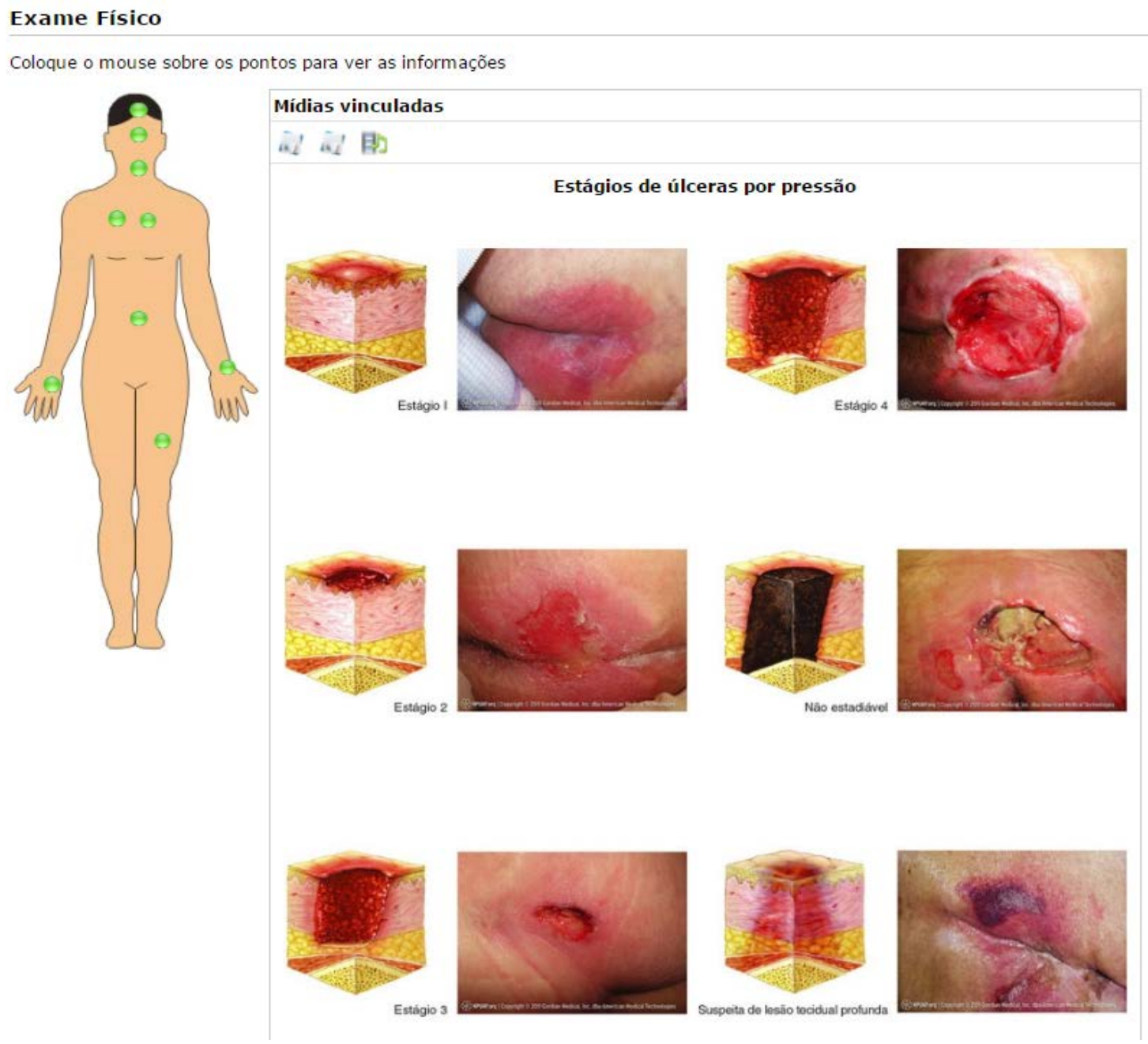

Figura 5 - Utilização de imagens nas informações do exame físico (2) Fonte: SIACC (2016)

Os exercícios de fixação de conteúdo (Figura 6) são de múltipla escolha, elaborados pelas próprias bolsistas ou retirados de provas de concursos públicos brasileiros para o cargo de enfermeiro em diferentes serviços de saúde. Os exercícios de fixação têm enfoque na fisiopatologia e tratamento das úlceras por pressão, além de abordar aspectos como levantamento de problemas de enfermagem e diagnósticos de enfermagem prioritários para o paciente em estudo. $\mathrm{O}$ aluno pode responder às questões e receber a correção imediata do exercício, com justificativas descritas em cada alternativa. Dessa forma, o discente recebe um feedback rápido e objetivo sobre o seu desempenho no exercício em questão, sendo ativo no seu processo de aprendizagem e gerando o conhecimento a partir de seus próprios erros e acertos.

As respostas dos alunos influenciam o desfecho do caso clínico, ou seja, a tomada de decisões no decorrer do caso exerce impacto na situação final do paciente simulado. Por exemplo, a escolha de cuidados inadequados para o tratamento das feridas do paciente em estudo poderia significar a piora no seu quadro clínico. 


\section{Pergunta}

(Prefeitura de Porto Velho-RO/Consulplan/2012) Com relação às úlceras por pressão é INCORRETO afirmar que:

A) a) alterações relacionadas à idade fazem com que a pele dos idosos seja menos propensa a traumas devido à pressão e ao atrito.

B) b) contril

C) c) cliente

E) e) a Esca pressão é INCORRETO afirmar que:

Q. (UFPA-PA/20

cicatrização n

tecnologias que 0 envolvidos no pri correto afirmar:

A) a) $O$ algir

B) b) Ao tra

impede a oxige

C) c) Ao util

produto.

D) d) Nas úl

E) e) Ao rea

atuação da en

(SES-PI/NUC

tecido de gran

cicatrização mais

posteriormente

esões traumátic corretas apenas:

A) a) $1,2 \mathrm{e}$

B) $\circ$ b) 2,3,

C) c) 2,3 e

D) d) $1,2,3$ e 5

E) e) 1 e 4

D) d) a pres Etapa: (Prefeitura de Porto Velho-Ro/Consulplan/2012) Com relação às úlceras por

a) alternações relacionadas à idade

Gabarito Você

a) alterações relacionadas à idade fazem com que a pele dos idosos seja menos propensa a traumas devido à mabarito pressão e ao atrito.

\section{pressão.}

ão de úlceras.

(2)

as por pressão.

oporcionam a

liado a novas

tipos de tecidos

5 com feridas, é

Incorreto. As alterações relacionadas à idade fazem com que a pele dos idosos

seja mais propensa a traumas devido à pressão e ao atrito.

b) contribuem para o seu

desenvolvimento a intensidade da

pressấo a duração da pressão e a

tolerância do tecido.

c) clientes incapazes de mudar de

posição de forma independente estão

em risco de desenvolvimento de

úlceras por pressão.

d) a presença e a duração da

umidade sobre a pele (fezes, urina,

drenagem de feridas) aumentam o

risco de formação de úlceras. cobertura secundária

ntes da aplicação do

para não realçar a

Ver detalhes

rimeira intenção, o da intenção produz

la aberta e

queimaduras,

da e mole. Estão

Figura 6 - Exercícios de fixação com verificação de respostas

Fonte: SIACC (2016)

A seção de exames complementares (Figura 7) permite que o aluno solicite os exames que gostaria de consultar para realizar o levantamento de problemas e diagnósticos de enfermagem, simulando o processo de tomada de decisões frequente na prática clínica.

Resultados dos exames solicitados

\section{Paciente realiza HGT em jejum pela manhã. - resultado}

Resultados do exame

$250 \mathrm{mg} / \mathrm{dl}$

Paciente realiza HGT à noite, após a janta - resultado

Resultados do exame

$255 \mathrm{mg} / \mathrm{dl}$

Hemograma - resultado

Resultados do exame

HGB - Hemoglobina $\quad 6,6 \mathrm{~g} / \mathrm{dl}$

WBG - Leucócitos $\quad 6520 \mathrm{u} / \mathrm{l}$

Plaquetas $10,5 \times 10^{3} / \mathrm{mm}^{3}$

HCT - Hematócrito $\quad 20 \%$

VCM - Volume corpuscular médio $70 \mathrm{fL}$

Neutrófilo segmentado $\quad 1,9 \%$

Eosinófilos $\quad 2,1 \%$

Neutrófilos bastonetes $\quad 71 \%$

Basófilos $\quad 0,1 \%$

Linfócitos $\quad 13,7 \%$

Monócitos $\quad 11,2 \%$

Figura 7 - Exames complementares

Fonte: SIACC (2016) 
A elaboração do caso clínico nesse formato permite que o aluno desenvolva as habilidades de pensamento crítico, utilizando a sistematização da assistência de enfermagem (SAE), definida como "uma metodologia científica de que o profissional enfermeiro dispõe para aplicar seus conhecimentos técnico-científicos e humanos na assistência aos pacientes" ${ }^{15}$. A SAE organiza o trabalho do enfermeiro quanto ao método, pessoal e instrumentos, de forma que seja possível operacionalizar o processo de enfermagem (PE).

O PE é organizado em: coleta de dados - anamnese e exame físico, diagnóstico de enfermagem, planejamento de enfermagem, resultados e intervenções de enfermagem, implementação, e avaliação de enfermagem.

No decorrer do caso, o discente escolhe os diagnósticos de enfermagem pertinentes ao paciente e os resultados e intervenções correspondentes, facilitando a integração entre os conhecimentos de semiologia e semiotécnica e a sua inserção nas etapas do processo.

A partir da leitura das informações da anamnese, exame físico e exames complementares, espera-se que o estudante esteja apto a identificar os problemas de enfermagem (Figura 8) no caso em estudo e levantar os diagnósticos (Figura 9), resultados e intervenções de enfermagem, que para sua formulação exigem a utilização de taxonomias próprias da enfermagem.

\section{Pergunta}

Q A partir da leitura da história da paciente, elenque os Problemas de Enfermagem neste caso.

A) Incontinência Urinária

B) Dor

C) $\square$ Úlceras

$\checkmark$ Imobilidade

E) $\square$ Confusão

F) Estrutura familiar desorganizada

G) $\square$ Negligência

H) Mobilidade limitada

Figura 8 - Problemas de enfermagem

Fonte: SIACC (2016)

\section{Diagnósticos de Enfermagem}

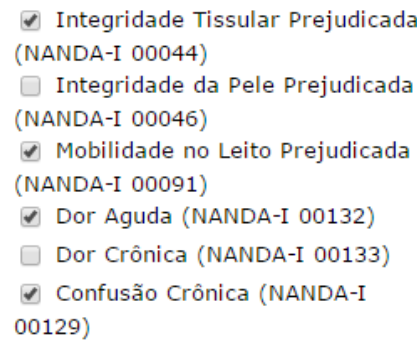

Figura 9 - Diagnósticos de enfermagem de acordo com a NANDA-I

Fonte: SIACC (2016)

Seções de conteúdo estão distribuídas no decorrer do caso clínico, abordando principalmente a avaliação de feridas, as formas de prevenção e o tratamento de úlceras por pressão, através da disponibilização de vídeos, imagens, websites, artigos científicos e manuais. O fornecimento de informações científicas atualizadas é a base da construção de uma prática profissional baseada em evidências, maximizando os benefícios e minimizando riscos e intervenções desnecessárias aos pacientes.

O caso clínico é finalizado com as opções de tratamento e o consequente desfecho do caso. Na seção de tratamento, são disponibilizadas diversas opções de curativos para as diferentes úlceras por pressão 
apresentadas pela paciente em estudo. Antes de escolher as opções, o aluno pode ainda clicar nas informações complementares disponíveis em cada alternativa com a explicação da forma de utilização e mecanismo de ação de cada curativo proposto. Essas informações reforçam o conteúdo já disponibilizado anteriormente no caso, de forma que o discente resgate os conhecimentos adquiridos no momento da resolução do exercício.

Dependendo da escolha da forma de tratamento, o desfecho será favorável ou desfavorável, proporcionando ao estudante um ambiente seguro para tomar decisões clínicas sem o risco de prejudicar um paciente real. No final, um relatório com toda a trajetória do aluno no caso e suas respostas é mostrado, de forma que ele possa entender o porquê do desfecho do tratamento do paciente em questão e possa retornar ao caso para responder novamente.

\section{Considerações finais}

A prática clínica exige a aquisição de habilidades complexas, o que causa ansiedade nos estudantes e que, se não for identificada ou manejada corretamente, pode afetar negativamente o seu desempenho ao realizar as suas tarefas ${ }^{16,17}$. O caso clínico virtual disponibilizado é um método de simulação que pode ser efetivo no que se refere à redução da ansiedade dos alunos, pois a possibilidade de errar e aprender com os erros permite que o aluno compreenda as consequências de suas escolhas em um ambiente seguro, sem impacto na vida do paciente como o observado quando o aluno passa diretamente das aulas teóricas para a atuação nos campos hospitalares e na comunidade.

A disponibilidade do caso clínico em uma plataforma virtual gratuita que pode ser acessada em qualquer tipo de dispositivo com internet se mostra favorável à sua implementação nas disciplinas dos currículos de enfermagem, além de permitir que o aluno gerencie o seu tempo e realize o caso no local e momento em que achar mais confortável e adequado conforme a sua rotina.

O caso clínico descrito neste artigo é piloto e, apesar de suas potencialidades, ainda há limitações no que se refere à programação do software e sustentabilidade do projeto; além de sua plataforma não ser específica para a enfermagem e sim destinada a todas as áreas da saúde, o que representa benefício por ser multidisciplinar, porém limitada em algumas etapas específicas do processo de enfermagem.

\section{Referências}

1. Freire P. Pedagogia da autonomia: saberes necessários à prática educativa. São Paulo: Paz e Terra; 1996.

2. Feuerwerker LCM. Gestão dos processos de mudanças na graduação em medicina. In: Marins JJN, Rego S, Lampert JB, Araújo JGC, colaboradores. Educação médica em transformação: instrumentos para a construção de novas realidades. São Paulo: Hucitec;2004. p. 17-39.

3. Souza CS, Iglesias AG, Pazin-Filho A. Estratégias inovadoras para métodos de ensino tradicionais: aspectos gerais. R Fac. Med. Ribeirão Preto. 2014 [citado em 2016 mar.10]; 47(3):284-92. Disponível em: http://revista.fmrp.usp.br/2014/vol47n3/6 Estrategias-inovadoras-para-metodos-de-ensinotradicionais-aspectos-gerais.pdf

4. Maris JJNE. A. Educação médica em transformação: instrumentos para a construção de novas realidades. São Paulo: Hucitec; 2004.

5. Brasil. Conselho Nacional de Educação. Câmara de Educação Superior. Resolução CNES/CES na 3 de 7 de novembro de 2001. Institui Diretrizes Curriculares Nacionais do Curso de Graduação em Enfermagem [online]. Diário Oficial da República Federativa da União, Brasília (DF); 2001. Disponível em: http:// portal.mec.gov.br/cne/arquivos/pdf/CES03.pdf 
6. Grossi MG, Kobayashi RMA. A construção de um ambiente virtual de aprendizagem para educação a distância: uma estratégia educativa em serviço. R Esc Enferm USP. 2013 [citado em 2016 mar. 10];47(3):756-60. Disponível em: http://www.scielo.br/pdf/reeusp/v47n3/0080-6234reeusp-47-3-00756.pdf

7. Barbosa SFF, Marin HF. Simulação baseada na web: uma ferramenta para o ensino de enfermagem em terapia intensiva. R Latino-Americana Enferm. 2009 [citado em 2016 mar. 10]; 17(1):[8 p.]. Disponível em: http://www.scielo.br/pdf/rlae/v17n1/pt 02.pdf

8. Bass J. Revolutionizing engineering science through simulation [Internet]. A report of the National Science Foundation Blue Ribbon Panel on Simulation-Based Engineering Science. [place unknown]; 2006. Disponível em: http://www.nsf.gov/pubs/reports/sbes final report.pdf

9. Stayt LC, Merriman C, Ricketts B, Morton S, Simpson T. Recognizing and managing a deteriorating patient: a randomized controlled trial investigating the effectiveness of clinical simulation in improving clinical performance in undergraduate nursing students. J AdvNurs. 2015 [cited 2016 Mar 10];71(11): 2563-74. doi: 10.1111/jan.12722

10. Bogossian FE, Cooper SJ, Cant R, Porter J, Forbes H. A trial of e-simulation of sudden patient deterioration (FIRST2ACT WEB) on student learning. Nurs Educ Today. 2015 [cited 2016 Mar 10];35(10): e36-e42. Disponível em: http://dx.doi.org/10.1016/j.nedt.2015.08.003

11. Kelly MA, Forber J, Conlon L, Roche M, Stasa H. Empowering the registered nurses of tomorrow: student's perspectives of a simulation experience for recognizing and managing a deteriorating patient. Nurs Educ Today. 2013 [cited 2016 Mar 10]; 34(5):724-29. Disponível em: http://dx.doi.org/10.1016/j. nedt.2013.08.014

12. Merriman CD, Stayt, LC, Ricketts B. Comparing the effectiveness of clinical simulation versus didactic methods to teach undergraduate adult nursing students to recognize and assess the deteriorating patient. Clin Simul Nurs. 2014 [cited 2016 Mar 10];10(3):e119-e27. Disponível em: http://dx.doi. org/10.1016/j.ecns.2013.09.004

13. Wiley DA. Connecting learning objects to instructional theory: a definition, a methaphor anda a taxonomy [Internet]. In: Wiley DA, editor. The instructional use of learning objets. [place unknown]: Agency for Instructional Technology; 2001[cited 2016 Mar 10]. Disponível em: http://www.reusability. org/read/

14. Sebastiani RL, Zago MK, Mantovani A, Marta RB, Bruno R, Dahmer A et al.Ferramenta de autoria para construção de casos clínicos interativos para educação médica [Internet]. In: Anais do $32^{\circ}$ Congresso da Sociedade Brasileira de Computação; 2012 July 16-19; Curitiba: UFPR; 2012. Disponível em: http:// www.imago.ufpr.br/csbc2012/anais csbc/eventos/wim/artigos/WIM2012\%20

15. Tannure MC, Pinheiro AM. Sistematização da assistência de enfermagem: guia prático. 2. ed. Rio de Janeiro: Guanabara Koogan; 2010.

16. Hutchinson TL, Goodin, HJ. Nursing student anxiety as a context for teaching/learning. J Holist Nurs. 2013 [cited 2016 Mar 10];31(1);19-24. Disponível em: http://jhn.sagepub.com

17. Melincavage SM. Student nurses' experiences of anxiety in the clinical setting. Nurs EduToday. 2011[cited 2016 Mar 10];31(8):785-89. Disponível em: doi:10.1016/j.nedt.2011.05.007. 